vertrat die Auffassung, dass zwischen Leninismus und Stalinismus getrennt werden müsse, sodass die konkrete politische Herrschaftspraxis der Bolschewiki nach der Oktoberrevolution unter Lenin nicht substanziell in den Blick genommen wurde. Daraus folgte für den Monat, dass die konkrete Terrorpolitik unter Lenin im Großen und Ganzen nicht im gleichen Maße thematisiert wurde wie die unter Stalin. Folglich liegt mit Blick auf die Beschreibung und Analyse des sowjetischen Kommunismus der Schwerpunkt auf dem Stalinismus (Kap. IV.2.2 bis IV.2.5) und dem Nachstalinismus (Kap. IV.2.6 bis IV.2.8). Das zeitliche Ende bildet in diesem Zusammenhang der XXI. Parteitag der KPdSU Anfang des Jahres 1959, in dessen Verlauf Chruschtschows »Alleinherrschaft« (Herbert Ritvo) institutionell installiert wurde. Den Abschluss dieses Kapitels bildet das »analytische Resümee« (Kap. IV.2.9), in dem der Versuch einer grundsätzlichen Einschätzung und Bewertung der Analyse des sowjetischen Kommunismus im Monat unternommen wird.

Worauf in diesem Kapitel aus Raumgründen nicht ausdrücklich eingegangen werden kann, ist der Themenkomplex über die Faszinationskraft, die der Kommunismus in der ersten Hälfte des 20 . Jahrhunderts auf zahlreiche Intellektuelle, zumal auf bürgerliche Intellektuelle in den westlichen kapitalistischen Staaten, ausübte, obwohl dies im Monat eine zentrale Bedeutung besaß. Auch vor dem Hintergrund der Tatsache, dass zahlreiche Autoren vor der Gründung dieses Periodikums selber einmal Mitglied in einer nationalen kommunistischen Partei (vor allem Deutschlands, Frankreichs und Italiens) waren, rückte in vielen Beiträgen die Frage der »unverbrüchlichen Treue« - trotz zum Beispiel eigener Erfahrungen oder Meldungen über die Moskauer Prozesse und den stalinistischen Terror in den 1930er-Jahren sowie des Hitler-Stalin-Paktes - zur Sowjetunion und dem in den allermeisten Fällen auch religiös konnotierten Glauben an die kommunistische Idee sowie der anschließende gemeinhin »schmerzvolle« Desillussionierungsprozess und endgültige »Bruch« ins Blickfeld der vielleicht international bekannteste Renegat Arthur Koestler steht hier pars pro toto. ${ }^{356}$

\title{
2.2 Die Russische Revolution als Genesis der kommunistischen Gewaltherrschaft
}

Als die bolschewistische Partei unter der Führung von Lenin im Oktober 1917 in Russland die politische Macht ergriff, waren sie der felsenfesten Überzeugung, dass eine "neue Zeit« anbrechen würde. Die Bolschewiki glaubten daran, als Vollstrecker historischer Gesetze eine radikal neue gesellschaftliche Ordnung Wirklichkeit werden zu lassen, die letzten Endes mit dem kapitalistischen System in der ganzen Welt Tabula rasa macht und den »neuen Menschen« hervorbringt. Entsprechend »der« Staats- und Revolutionstheorie von Lenin sollte ihrer festen Überzeugung nach den bisherigen Formen des politischen und gesellschaftlichen Lebens ein Ende bereitet werden, damit auf der Grundlage einer modifizierten Theorie des Marxismus zuerst die russischen Verhältnisse verändert werden. Das neue Staatswesen besaß Lenins Theorie nach einen provisorischen Charakter und sollte, bis durch den Sieg der Weltrevolution der Übergang zur kommunistischen Gesellschaft hergestellt würde, eine »Diktatur

356 Siehe von den zahlreichen Beiträgen im Monat exemplarisch besonders die Reihe Der Cott, der keiner warbzw. Ein Cott hat versagt. 
des Proletariats« sein, wobei der Wille des Proletariats von einer Partei, sprich den Bolschewiki, repräsentiert werden sollte. Dass sich daraus in der Sowjetunion die Herrschaft einer Partei bzw. ein staatliches Regime entwickeln würde, das in seiner repressivsten, d. h. totalitären Phase unter Stalin verantwortlich war für Millionen von Toten, für Massenverhaftungen durch den Geheimdienstapparat, für ein das ganze Land überzogenes Konzentrations- und Arbeitslagersystem, war indes zum Zeitpunkt der mehr einem Putsch gleichkommenden Machteroberung durch die Bolschewiki unter der Führung Lenins offen. Die Entwicklung nach der Revolution mit der Konstituierung des bolschewistischen Regimes, des Leninismus, und der Weiterentwicklung zum totalitären Herrschaftssystem unter Stalin, des Stalinismus, verlief indes keineswegs geradlinig. ${ }^{357}$ Überhaupt war es ein historisch-politisch offener Prozess, der dazu führte, dass die Bolschewiki mit der Oktoberrevolution 1917 Erfolg hatten. ${ }^{358}$

Die Bolschewiki standen in der Tradition der russischen Sozialdemokraten und Sozialrevolutionäre, die in das 19. Jahrhundert zurückreichte und die ohne die Rolle der Intellektuellen kaum zu verstehen war. Die führenden Parteifunktionäre - Lenin steht hier pars pro toto - standen zweifelsohne in der Tradition des russischen Intellektuellen des 19. Jahrhunderts und teilten im Großen und Ganzen die in dieser Zeit zum Ausdruck gebrachten politischen Ideen und Grundüberzeugungen, die in erster Linie eine Mischung aus einem (extremen) russischen Nationalismus, panslawistischen Imperialismus und Feindschaft gegen den »Westen«, anders gesagt eine Feindschaft gegen den Liberalismus der westlichen Gesellschaft, darstellten. Vor dem Hintergrund der Ablehnung der bestehenden Gesellschaftsordnung entwickelte sich hieraus die Vorstellung, der Glaube eines "russischen Sendungsbewusstseins«, wobei Fjodor Michailowitsch Dostojewski den Prototypen des russischen Intellektuellen des 19. Jahrhunderts darstellte. ${ }^{359}$ Die Bolschewiki waren, angesichts der herrschenden sozialen und politischen Bedingungen im Zarenreich, ${ }^{360}$ beseelt von dem unbedingten Glauben an den Sturz der bestehenden (vor-)kapitalistischen Gesellschaftsordnung und meinten sich des Rückhalts bei den russischen Bauern und bei dem sich entwickelnden Proletariat sicher. Dabei verkannte die russische Intelligenzija ihre isolierte Situation, denn die Bevölkerung wollte von Befreiung, von Emanzipation aus den zum Teil katastrophalen Lebensumständen nichts wissen. In Anbetracht ihrer Marginalisierung, eindeutiger Feindbilder und permanenter polizeilicher Überwachung und Verfolgung entwickelte sich bei den Mitgliedern der kleinen revolutionären Gruppen eine Verhaltensweise heraus, die man aus religiösen Sekten kannte und deren Charakteristikum vor allem war, dass sich die an ihre "Mission« glaubenden Mitglieder untereinander auf das Genaueste überwachten. Eingedenk der Gefahr einer Gruppenspaltung wurde jedes abweichende Verhalten und jede abweichende Meinung eines

357 Siehe Franz Borkenau, Zwei Revolutionäre, in: Der Monat 3 (1950), H. 25, S. 19-29, hier S. 22 (Rezension). Die Angaben zu dem Buch erfolgen hier - wie auch im Folgenden - nach den redaktionellen Mitteilungen des Monat. In der Regel wurde hierbei das Erscheinungsjahr des jeweiligen Buches nicht mitangegeben.

Vgl. Sidney Hook, Rußland im Mondenschein. Bemerkungen zu einem neuen Buch über die Sowjetunion, in: Der Monat 4 (1952), H. 44, S. 172-178, hier S. 174 (Rezension).

359 Vgl. Hans Kohn, Dostojewski. Ein politisches Porträt, in: Der Monat 2 (1949), H. 13, S. $78-88$ (Teil I), sowie H. 14, S. 187-192 (Teil II).

360 Vgl. Hans Kohn, Rußland vor Lenin, in: Der Monat 6 (1954), H. 67, S. 75 f. (Rezension). 
Mitglieds zur Kenntnis genommen. Vor diesem Hintergrund entstand ein Typus des russischen Revolutionärs, deren radikalste Verkörperung Sergei Netschajew war. Seine historische Originalität beruhte nicht allein darauf, dass er entsprechend einer »revolutionären Moral« handelte und demzufolge auch jedwedes Verbrechen vom Diebstahl bis zum Mord für legitim hielt, sondern vor allem darauf, dass er auch bereit war, »seine Prinzipien mit der gleichen Bereitwilligkeit auf Freund und Feind« anzuwenden. ${ }^{361}$

Auf dem zweiten Parteitag der russischen sozialdemokratischen Partei im Jahre 1903 kam es vor allem infolge der Frage der Parteiorganisation zur Spaltung in zwei Gruppen, in eine größere Mehrheitspartei unter Lenin (Bolschewiki) und eine Minderheitspartei (Menschewiki). Diese Spaltung sollte auch nach der ersten Russischen Revolution von 1905 nicht wieder rückgängig gemacht werden und war ein Grund für erbitterte Auseinandersetzungen. Denn die bolschewistische Parteiorganisation stand im Unterschied zu den Menschewiki der Idee einer breiten Massenorganisation nach dem Muster der deutschen Sozialdemokraten feindlich gegenüber und favorisierte das Konzept der Partei als einer Avantgarde des Proletariats. Lenin entwarf im Jahre 1902 in seiner Schrift Was tun? das zentrale Organisationsschema, nach dem dann die bolschewistische Bewegung auch aufgebaut wurde. Die Bauern und Arbeiter, d.h. die »Massen«, sollten im Hinblick auf die "proletarische Revolution« von einer kleinen verschworenen Gruppe, von einem engen Zirkel von bolschewistischen Berufsrevolutionären geführt werden. ${ }^{362}$

Die Konstituierung des bolschewistischen Regimes unter Lenin nach der Oktoberrevolution 1917 wäre allerdings ohne den Ausbruch und den Verlauf des Ersten Weltkrieges kaum möglich gewesen und erfolgte keineswegs zwangsläufig. Die frühen militärischen Niederlagen der russischen Armeen lösten eine wirtschaftliche Krise aus, die zu Hungersnöten in der Bevölkerung des gesamten zaristischen Landes führte. Streiks und Aufstände der städtischen und vor allem der ländlichen Bevölkerung waren die Folge. Ganze Scharen von hungernden Menschen zogen durch das Land und widersetzten sich der autokratischen Staatsgewalt. Da der Krieg im Laufe der Jahre große Opfer an Menschenleben gekostet hatte, verweigerten mehr und mehr Soldaten den Dienst an der Waffe. Die sozialen Nöte korrespondierten mit einer sich immer schneller ausbreitenden politischen Unzufriedenheit in der russischen Bevölkerung. Die kaum noch zu kontrollierenden frustrierten Massen entwickelten sich zu einer ernsthaften Gefahr für das mit polizeistaatlichen Methoden herrschende Regime des Zaren Nikolaus II. Die im Zuge der Revolte im Jahre 1905 erzwungene bürgerliche Verfassung, die unter anderem das Wahlrecht vorsah, wurde sukzessive beschnitten. Ohne das die Bolschewiki - Lenin und andere Führer der Partei befanden sich zu diesem Zeitpunkt im Exil oder in Haft - auf die Massen einen nennenswerten Einfluss hatten, geschweige denn imstande waren, ihnen ihren »Willen« aufzuzwingen, erhoben sich Soldaten und die Bevölkerung in Petrograd im März 1917 und besetzten, ohne das Blut vergossen wurde, die Duma, d.h. das Parlament mit »beschränkten Befugnissen«. Kurz darauf schwappte die revolutionäre Stimmung auch auf die Bevölkerung Moskaus über, die mit den gegen sie aufgestellten militärischen Truppen

361 Edward Hallett Carr, Die Affäre Netschajew. Ein Kapitel russischer Revolutionsgeschichte, in: Der Monat 2 (1950), H. 24, S. 535-544, hier S. 535.

362 Franz Borkenau, Zwei Revolutionäre, in: Der Monat 3 (1950), H. 25, S. 23. 
keine Probleme hatte, und brachte in kürzester Zeit ohne Blutvergießen das Regime des verhassten Zaren zum Einsturz, und es wurde eine sogenannte demokratische provisorische Regierung gebildet. "Das Kabinett«, so der amerikanische Journalist und Historiker Boris Shub,

das zumeist aus Liberalen bestand, wurde vom Duma-Komitee und dem Exekutiv-Komitee des neugebildeten Petrograder Sowjets der Arbeiter- und Soldatendeputierten ausgewählt - einer spontan entstandenen revolutionären Versammlung, deren Führer bis zum Oktober Demokraten und keine Kommunisten waren. Als Verbindungsmann zwischen der Duma und dem Sowjet wurde Kerenski Justizminister und später Ministerpräsident. ${ }^{363}$

$\mathrm{Zu}$ den ersten Maßnahmen der provisorischen Regierung gehörte es, per Dekret die politischen Gefangenen aus den Gefängnissen zu entlassen und demokratische Grundrechte wie Rede-, Presse- und Versammlungsfreiheit zu garantieren sowie soziale, religiöse oder aus der Zugehörigkeit zu einer nationalen Minderheit resultierende Benachteiligungen aufzuheben. Um den Bruch mit dem zaristischen Regime $\mathrm{zu}$ verdeutlichen, wurde die in der Bevölkerung verhasste Geheimpolizei sowie die Todesstrafe abgeschafft und die provisorische Regierung kündigte die Wahl einer konstituierenden Versammlung unter allgemeinem Wahlrecht an. An der sogenannten demokratischen Revolution im März 1917 spielten die Bolschewiki keineswegs eine führende Rolle, zumal die Partei alles andere als eine disziplinierte Organisation darstellte. ${ }^{364}$

Lenin kehrte nach dem Sturz der Zarenherrschaft aus seinem insgesamt zehn Jahre andauernden Exil aus der Schweiz nach Russland zurück. Seiner Strategie aus dem Jahre 1914 zufolge sollte der Erste Weltkrieg, der qua kommunistischer Ideologie ein »imperialistischer Krieg« war, in einen Bürgerkrieg verwandelt werden, um das zentrale Ziel, die politische Macht zu ergreifen, die "Diktatur des Proletariats« zu errichten, und die klassenlose Gesellschaft Wirklichkeit werden zu lassen. Lenin hatte den Ausbruch des Ersten Weltkrieges keineswegs beklagt und interpretierte ihn gewissermaßen als Vorspiel der Revolution. Getreu dem Motto Netschajews war dem Berufsrevolutionär Lenin zur Erreichung seines Zieles jedes Mittel recht. Die »neue« Regierung geriet auch durch ihn unter Druck, weil Lenin sich für einen Friedensvertrag mit dem »imperialistischen Deutschland« (Ruth Fischer) aussprach. Lenin erkannte die Möglichkeit, mit einer defätistischen Propaganda für den »Frieden« die kriegsmüden russischen Truppen aufzufordern, die Schützengräben zu verlassen und in die geliebte Heimat zurückzukehren, damit sie ihren Anteil an Grund und Boden in Besitz nehmen konnten, ohne auf eine gesetzliche Regelung zu warten. ${ }^{365}$ Daraufhin desertierten in den Monaten April bis Oktober 1917 Hunderttausende Soldaten - dem sich auch Offiziere anschlossen - und kehrten in ihre Dörfer zurück, um zum einen bei der Landverteilung dabei zu sein und zum anderen um bei der Ernte zu helfen. Wäre die »Kerenski-Regierung« in diesen Monaten unter anderem aufgrund der Propagandatätigkeiten entschieden gegen Lenin und gegen die anderen Führer der Bol-

363 Boris Shub, Die vergessene Revolution, in: Der Monat 4 (1952), H. 42, S. 654-656, hier S. 655.

364 Vgl. hierzu Harald Hurwitz, Die verfälschte Revolution, in: Der Monat 4 (1952), H. 44, S. 216-218.

365 Boris Shub, Die vergessene Revolution, in: Der Monat 4 (1952), H. 42, S. 655. 
schewiki vorgegangen, wäre es möglicherweise nicht zur siegreichen Machtergreifung im Oktober gekommen. ${ }^{366}$

Die provisorische Regierung hatte allerdings keine Möglichkeit, dem Auseinanderbrechen seiner Armee etwas Entscheidendes entgegenzusetzen. Auch die sogenannte Kerenskioffensive erwies sich letzten Endes als erfolglos. Parallel zum Zerfall der Armee sah sich die neue Regierung aufgrund der weiterhin existierenden gravierenden Versorgungsprobleme im ganzen Land, die mit einer galoppierenden Inflation korrespondierte und das wirtschaftliche Chaos auf die Spitze trieb, in den Städten mit einer Welle von Streiks der Arbeiter konfrontiert. Die sich auch durch die heftigen Zusammenstöße mit der "Staatsgewalt«, d.h. der Miliz, sukzessive radikalisierende Bewegung stellte die zentrale politische Forderung, die Betriebe unter die Kontrolle der Arbeiter zu stellen. Im ganzen Land, nicht nur in den beiden großen Städten Moskau und Petrograd, bildeten sich Arbeiter- und Soldatenräte, d. h. die Sowjets, die der neuen Regierung den Kampf ansagten. Zudem tobte auf den Straßen die Gewalt und es kam vor allem aufgrund der sozialen und wirtschaftlichen Situation im ganzen Land zu Plünderungen, sodass die Kerenskiregierung, die nur noch über eine kleine Milizeinheit verfügte und der mithin kein eigentliches Machtinstrument zur Verfügung stand, immer mehr unter Beschuss geriet und die politische Ordnung kaum noch aufrechterhalten konnte. Die Bolschewiki erkannten angesichts dieser Situation, als die »Macht auf der Straße« lag, ihre Chance. Vor dem Hintergrund der Parole Lenins »Alle Macht den Sowjets« gelang es Einfluss auf die Arbeiter und Soldaten zu nehmen, und den Bauern wurde versprochen, ihre Forderungen zu erfüllen. Gleichwohl waren es keineswegs die »Massen«, die den Aufstand wagten und die die Oktoberrevolution herbeiführten. Entscheidend war in diesem Zusammenhang der »feste Wille« Lenins, die politische Macht zu erobern, sodass letzten Endes eine »Handvoll«, d.h. ein paar tausend entschlossener, bewaffneter Einheiten der Bolschewiki genügten - die aber zweifelsohne insbesondere mit der Zustimmung von Millionen von Bauern und Soldaten, weniger der Arbeiter -, im Oktober 1917 »ihren Staatsstreich in Szene« zu setzen. ${ }^{367}$

Nach der erfolgreichen Machteroberung war die bolschewistische Partei unter der Führung Lenins fest entschlossen, die Macht mit allen Mitteln zu verteidigen und vor dem Hintergrund der marxistisch-leninistischen Ideologie die "Diktatur des Proletariats « in Russland zu errichten sowie im Zuge eines notwendigen historischen Prozesses zuerst die klassenlose kommunistische Gesellschaft in Russland und danach in der ganzen Welt herbeizuführen. Lenins Utopie war die Weltrevolution. Seiner politischen Weltanschauung nach, die freilich von den führenden Parteimitgliedern geteilt wurde, bestand die Weltpolitik aus einem unversöhnlichen Kampf zwischen Bolschewismus respektive Kommunismus und Kapitalismus. ${ }^{368}$ Anders formuliert: Die führenden Vertreter des bolschewistischen Regimes waren der festen ideologischen Überzeugung, dass zwischen der russischen bzw. sowjetischen Welt und der nichtsowjetischen Welt ein grundsätzlich unüberbrückbarer Gegensatz besteht, und des-

366 Sidney Hook, Rußland im Mondenschein. Bemerkungen zu einem neuen Buch über die Sowjetunion, in: Der Monat 4 (1952), H. 44, S. 172-178, hier S. 174 (Rezension).

367 Boris Shub, Die vergessene Revolution, in: Der Monat 4 (1952), H. 42, S. 656.

368 David Shub, Lenins letzte Tage. Aus einer neuen kritischen Biographie, in: Der Monat 1 (1948), H. 3, S. $37-48$, hier S. 41. 
halb hingen Lenin und auch beispielsweise sein späterer Nachfolger, also Stalin, dem Glauben an, dass die weltweite Ausbreitung des »sowjetischen Systems« die entscheidende Voraussetzung zur eigenen Sicherheit darstellt. Lenin und seine Anhänger betrachteten die Russische Revolution als das erste Stadium eines weltweiten Prozesses, an dessen Ende entsprechend einer historischen Notwendigkeit die siegreiche Weltrevolution steht. Entsprechend der marxistischen Ideologie war das sogenannte Endziel, das allen kommunistischen Bestrebungen ihren Sinn gab, die Schaffung einer klassenlosen Gesellschaft im »Weltmaßstab« (Löwenthal). Hieraus ergab sich das Konzept der "permanenten Revolution«, das gewissermaßen von Karl Marx vorgedacht wurde, als er die Hoffnung hatte, dass die bürgerlich-demokratischen Revolutionen in Europa in den Jahren 1848 bis 1850, "nachdem sie einmal die Arbeiterklassen der höchstentwickelten Länder in Bewegung gesetzt hatte, in ihrer zweiten Phase zu einer proletarischen Revolution führen«, so Löwenthal in seinem Beitrag Stalins Vermächtnis. Zur Interpretation seiner letzten Schrift im Monat aus dem Jahre $1953 .{ }^{369}$ Das Konzept der »Permanenten Revolution« wurde von Leo Trotzki, der neben Lenin die wichtigste Rolle bei der Machtergreifung der Bolschewiki spielte, aus den Erfahrungen der Russischen Revolution von 1905 entwickelt. Während des Ersten Weltkrieges tauchte der Gedanke bereits implizit bei Lenin wieder auf und sollte in den unmittelbar folgenden Jahren seine politischen Ziele bestimmen und stellte ein zentrales Element in der kommunistischen Theorie dar, denn: »Lenin erwartete damals, die bolschewistische Machtergreifung im rückständigen Rußland werde der Funke sein, der in den vom Krieg erschütterten fortgeschrittenen Industrieländern die Flamme von Massenaufständen entzünden werde. ${ }^{370}$

Gleichwohl galt es erst einmal, die gewonnene politische Macht zu sichern und die Revolution gegen ihre »Feinde« zu verteidigen, d.h. gegen den Klassenfeind, gegen den Adel und gegen die »Bourgeoisie« des Landes und gegen die Mitglieder anderer Parteien, wie den Liberalen, den Menschewiki oder den Sozialrevolutionären, sowie gegen die Anarchisten. Da man sich angesichts der bolschewistischen Ideologie bis zur Herstellung der klassenlosen Gesellschaft in einem dauernden Kriegszustand befand, ${ }^{371}$ waren nach Auffassung von Lenin und seiner Mitkämpfer die Unterdrückung aller bürgerlichen Rechte und die Ausübung von Gewalt ein legitimes Mittel. Der »Lenin-Biograph « David Shub schrieb in diesem Zusammenhang in der Veröffentlichung Lenins letzte Tage dazu im Monat:

Die marxistische Theorie übermäßig vereinfachend, verneinte er schließlich jede Cültigkeit der von der »Bourgeoisi«-Demokratie gewährten Rechte. [...] In der vorrevolutionären Zeit hat Lenin nicht immer das Versammlungsrecht als kapitalistischen Schwindel abgetan, sondern erst nach der Einführung des Sowjetsystems, als dieses sich Schritt für Schritt dazu gedrängt sah, jede politische Freiheit auszurotten, um seine Macht zu konsolidieren und festzuhalten. ${ }^{372}$

369 Richard Löwenthal, Stalins Vermächtnis. Zur Interpretation seiner letzten Schrift, in: Der Monat 3 (1953), H. 55, S. 16-25, hier S. 16.

370 Ebd., S. 17.

371 Vgl. Burnham, Vom Wesen des Kommunismus, S. 27.

372 David Shub, Lenins letzte Tage. Aus einer neuen kritischen Biographie, in: Der Monat 1 (1948), H. 3 , S. 41. 
Vor dem Hintergrund der marxistischen Utopie ergab sich nach Auffassung der bolschewistischen Parteiführer die "Diktatur des Proletariats « mit geschichtlicher Notwendigkeit. Da zudem »echte Demokratie« erst in der herzustellenden »klassenlosen« Gesellschaft, also im Kommunismus, verwirklicht werden könne, betrachtete es Lenin als »sein« historisches Recht, mit Gewalt gegen die Verfassungsgebende Versammlung, in der die Bolschewiki bei den Wahlen die Mehrheit verfehlten, vorzugehen. Lenin war sich über die Bedeutung im Klaren, denn die Auflösung der Verfassungsgebenden Versammlung bedeutete das Ende der »demokratischen Idee« und die Gewöhnung und Etablierung diktatorischer bzw. prätotalitärer Methoden. Die historisch-politischen Implikationen erkannte damals Maxim Gorki, der in einem Brief Anfang Januar 1918 an seinen »alten Freund« Lenin schrieb:

Seit beinahe einem Jahrhundert haben die Besten Rußlands von diesem Traum geträumt. Die Verfassungsgebende Versammlung hat ihnen vorgeschwebt als ein politisches Instrument, das der russischen Demokratie eine Celegenheit zur freien Äußerung ihres Willens zu bieten vermag. Tausende aus den Schichten der Intelligenz, Zehntausende von Arbeitern und Bauern sind im Cefängnis gestorben, sind erhängt worden, für diesen Traum. Ströme von Blut sind für diese heilige Idee vergossen worden. Und nun, da das Ziel erreicht ist und die Demokratie zu jubilieren anhebt, haben die »Volkskommissare« Befehl zum Schießen gegeben [...]. Ich frage die »Volkskommissare«, unter denen sich ehrliche, vernünftige Menschen befinden sollten, ob sie sich darüber klar sind, daß [...] sie die russische Demokratie unterdrücken und die Früchte der Revolution vernichten. ${ }^{373}$

Die Führung der Bolschewiki sah sich alsbald mit der »Konterrevolution« konfrontiert, die einen blutigen und lange andauernden Bürgerkrieg zur Folge hatte und die eine Spirale der Gewalt in Gang setzte. Dem »weißen Terror« setzte das neue Regime den »roten Terror« entgegen, der sich gegen alle »Feinde« der Revolution richten sollte. Mit der im Dezember 1917 in Petrograd ins Leben gerufenen Außerordentlichen Kommission zur Bekämpfung von Konterrevolution unter der Leitung des polnischen Kommunisten Feliks Dzierzynski schufen sich die Bolschewiki ein Instrument, um die sogenannte Konterrevolution zu bekämpfen. Der Geheimdienstapparat, sprich die Tscheka bzw. die spätere GPU, so die Auffassung der beiden ehemaligen russischen Sozialdemokraten David J. Dallin und Boris I. Nikolajewski,

wurde zunächst als eine vorläufige Einrichtung angesehen, die bald verschwinden und das ganze Cebiet anderen, liberalen Regierungsstellen überlassen würde; Todesstrafen waren desgleichen nur als eine »vorübergehende Notwendigkeit« gegen die »kriegerische Konterrevolution« ein Teil des neuen Rechts. Jeder bewaffnete Widerstand gegen das neue Regime hörte Anfang der zwanziger Jahre auf, aber die GPU fuhr fort, zu wachsen und sich auszudehnen. Sie erweiterte ihr eigenes Netz von Gefängnissen und Konzentrationslagern. Sie machte im Überfluß von ihrem Recht Gebrauch, Todesurteile auszusprechen und ihre eigenen Sprüche auszuführen. Sie überschattete bald 
das »liberale« Justizdepartment, und in diesem Sieg der GPU fanden die vorherrschenden Tendenzen und die neue Politik ihren klaren Ausdruck. ${ }^{374}$

Mit dem siegreichen Ende des Bürgerkrieges Anfang 1921 durch die »Rote Armee« unter der Leitung des Kriegskommissars Trotzki machte sich in der russischen Bevölkerung die Hoffnung breit, dass nicht nur der in Gang gekommene »rote Terror « ein Ende finden und die Tscheka abgeschafft würde, sondern dass sich das bolschewistische Regime insgesamt liberalisiert. Neben der Garantierung von demokratischen Freiheitsrechten sehnte man die Aufhebung des »Bürgerkriegssozialismus«, der die Verstaatlichung der Fabriken und die zentrale Regulierung des Handels beinhaltete, herbei. Allerorten war in der Bevölkerung das Bedürfnis vorhanden, dass sich endlich nach Jahren die rechtliche, soziale und wirtschaftliche Situation und vor allem die Versorgungslage des Landes verbessert, denn die Ernährungssituation war angesichts der Missernte des Jahres 1920, in deren Folge es zu einer Hungersnot im ganzen Land kam, außerordentlich schwierig. Insbesondere in der Bevölkerung auf dem Lande keimte die Hoffnung auf, dass die Beschlagnahmung ihres Getreides durch die "Getreidebeschaffungskommandos« (Shub) ein Ende findet. Nachdem im Februar 1921 in Petrograd Arbeiter in den Ausstand traten und öffentlich zum Streik aufriefen, reagierte das bolschewistische Regime mit eiserner Gewalt. Das Petrograder »Verteidigungs-« bzw. »Exekutiv-Komitee« unter dem Vorsitzenden Sinowjew verkündete das »außerordentliche Kriegsrechts « und ging entschlossen mit Einheiten der »Roten Armee« gegen den Protest vor. Im März desselben Jahres verlangten in Kronstadt, das in der unmittelbaren Nähe der ehemaligen »Hauptstadt« der ersten Russischen Revolution lag, Tausende von Arbeitern und Matrosen freie Wahlen der Sowjets, freie Gewerkschaften, Rede- und Pressefreiheiten, die Freilassung politischer Gefangener und die Abschaffung der Tscheka. Nachdem die Bolschewiki den Kronstädter Protest als »konterrevolutionären Aufstand« klassifizierte und verurteilte, wurde er durch Einheiten des russischen Militärs unter der Führung Trotzkis und des Generals Tuchatschewski sowie durch Tscheka-Einheiten blutig niedergeschlagen. In den folgenden Tagen kam durch die Tscheka eine regelrechte »Säuberungswelle« gegen die sogenannten Rebellen in Gang, in deren Folge unzählige Menschen bei »nächtlichen Massenerschießungen« getötet wurden. Auf die historisch-politische Bedeutung der Ereignisse im Jahre 1921 kam der russische Emigrant und Gegner des bolschewistischen Regimes Alexander Berkman zu sprechen. Bereits ein Jahr nach den Ereignissen schrieb er in einem Beitrag, den der Monat im Jahre 1951 veröffentlichte:

Mehrere Wochen lang waren die Petrograder Cefängnisse noch mit Kronstädter Cefangenen überfüllt. Jede Nacht wurden kleine Gruppen auf Befehl der Tscheka herausgeholt und verschwanden auf Nimmerwiedersehen. Die Cefängnisse und Konzentrationslager der eisigen Cebiete von Archangelsk und die Kerker im fernen Turkestan setzten dem Leben jener Männer ein Ende, die sich gegen die bolschewistische Büro-

374 Dallin/Nicolaevsky, Arbeiter oder Ausgebeutete, S. 66f.; hierbei handelte es sich um einen Teilauszug des Buches Forced Labor in Soviet Russia, vgl. hierzu Boris Shub, Lüge und Illusion, in: Der Monat 1 (1948), H. 1, S. 88-90 (Rezension). 
kratie erhoben und im März 1921 gewagt hatten, die Parole der Revolution von 1917 auszurufen: »Alle Macht den Räten! « ${ }^{375}$

Der »rote Terror « richtete sich in den ersten Jahren nach der bolschewistischen Machtergreifung also keineswegs nur gegen die »Klassenfeinde«. Von Anbeginn weitete er sich auch auf die Menschewiken, die Anarchisten und vor allem auf die Sozialrevolutionäre aus. ${ }^{376}$ Unter Lenin wurde, wie in einem Leserbrief im Monat zu erfahren war, gegen die führenden Vertreter der Sozialrevolutionären Partei im Jahre 1922 ein erster großer Moskauer Prozess - der gewissermaßen das Vorspiel der späteren Moskauer Schauprozesse darstellte - veranstaltet und aufgrund der Devise des Staatsanwaltes Krylenko, dass das Gericht ausschließlich dem Primat der sowjetischen Regierung unterliegt und den politischen Interessen der bolschewistischen Partei folgt, abgeurteilt. $^{377}$

Gleichwohl sollte sich der Terror auch gegen die sogenannte normale Bevölkerung insbesondere auf dem Lande, d.h. die Bauernschaft, richten. Auch wenn der eigentliche Terror zweifelsohne mit dem Beginn der Kollektivierung der Landwirtschaft bzw. der »Kollektivierungspolitik« unter Stalin schließlich im Jahre 1929 in Gang kam, ging das bolschewistische Regime bereits unter Lenin mit brutaler Gewalt gegen den Widerstand der Bauernschaft vor, die sich gegen die staatliche Requisition der Lebensmittel zur Wehr setzte. Die gegen ebendiese Politik gerichteten Aufstände vor allem in der Südukraine unter der Führung des Anarchisten Nestor Machno wurden von der "neuen« Regierung erbarmungslos niedergeschlagen. Hierbei ergab sich die Feindschaft gegen die Bauern, wie Michael Padev in einem ausführlichen Leserbrief im Monat schrieb, aus den genuinen Anschauungen von Lenin, die er in seiner 1908 erschienenen »klassischen Analyse « des Bauernstandes, Die Agrarfrage, zum Ausdruck brachte. Lenins Auffassung nach, so Padev, waren das Bauerntum bzw. die Bauern von ihm in drei verschiedene Gruppen unterschieden: Kleinbauer, mittlere Bauern und Großbauern (Kulaken): »die letzte kapitalistische Klasse«, und mithin hatte auch er keinerlei moralische und politische Bedenken, gegebenenfalls gegen sie vorzugehen. Die Kulaken wurden nicht als solche »bezeichnet und liquidiert, weil sie größere oder bessere Höfe« besaßen, sondern weil sie »die natürlichen Führer des Landvolkes« waren und sozusagen a priori als »Klassenfeind« apostrophiert wurden. Um sie zu »beseitigen«, mussten »sie physisch vernichtet werden «. ${ }^{378}$

Mit der Gründung der Kommunistischen Internationalen (Komintern), ein $\mathrm{Zu}$ sammenschluss der internationalen kommunistischen Parteien, schufen sich die Bolschewiki im Jahre 1919 eine Organisation, mit der sie glaubten, ihr Ziel, das kommunistische System der ganzen Welt aufzuzwingen, in die Praxis umzusetzen. Unter der

375 Alexander Berkman, Der Aufstand von Kronstadt. Zum 30. Jahrestag der ersten Revolution gegen den Bolschewismus, in: Der Monat 3 (1951), H. 30, S. 632-641, hier S. 641; hierbei handelte es sich um einen Aufsatz bzw. »Augenzeugenbericht«, der zum ersten Mal 1922 in Berlin erschien und, so die redaktionelle Mitteilung auf S. 666 im selben Heft, dem Sammelwerk Julien Steinberg, Verdict of Three Decades, New York o. J. entnommen wurde.

376 Vgl. David Shub, Lenins letzte Tage. Aus einer neuen kritischen Biographie, in: Der Monat 1 (1948), H. 3, S. 43 .

377 Siehe: Wie führt man Schauprozesse?, in: Der Monat 2 (1949), H. 14, S. 217.

378 Michael Padev, Die Bauern unter dem Sowjetsystem, in: Der Monat 4 (1951), H. 39, S. 327-329, hier S. 328 . 
Führung des Vertrauten Lenins, Grigori Sinowjew, sollte die internationale revolutionäre Arbeiterbewegung ausschließlich für die politischen Interessen Moskaus dienstbar gemacht werden. In seinem Beitrag über die Kominform nach dem Ende des Zweiten Weltkrieges, d.h. über die »Neue Komintern«, kam Borkenau auf den russischen Führungsanspruch innerhalb der »alten« Komintern zu sprechen. Im Monat schrieb er hierzu im Jahre 1949:

Der Keim der neuen Komintern war in der alten enthalten. Während Lenin die zukünftige Überlegenheit der industrialisierten Hauptländer des Westens anerkannte, bestand er auf der damaligen politischen Überlegenheit der russischen Bolschewiki über die entstehenden kommunistischen Parteien außerhalb Rußlands. Die Führung der Komintern lag von Anfang an in russischer Hand. Sieben Jahre lang war Gregori [sic !] Sinowjew, der kommunistische »Gauleiter« von Leningrad, ihr Vorsitzender. Auf ihn folgte 1926 Bucharin, auf diesen 1929 Molotow. ${ }^{379}$

Zweifelsohne war der »Keim« der sogenannten neuen Komintern in der »alten« enthalten. Gleichwohl gab es Unterschiede, auf die Borkenau zu Recht rekurrierte. So konstatierte er auch mit Blick auf die innerparteiliche Situation der Bolschewiki in der Sowjetunion:

\begin{abstract}
Aber die russische Führung der alten Komintern bedeutet etwas anderes als russische Führung der neuen. Die UdSSR war noch keineswegs ganz der totalitäre Staat, der sie heute ist. Bis 1921 gab es noch Oppositionsparteien, was freilich die Komintern wenig berührte. Viel wesentlicher war, daß es bis etwa 1926 innerhalb der Kommunistischen Partei Rußlands eine erhebliche, wenn auch langsam einschrumpfende Diskussionsfreiheit gab. Innerhalb der Partei bildeten sich immer wieder oppositionelle Richtungen, die bis zu einem gewissen Crade oppositionelle Parteien im Lande ersetzten. Diese Fraktionsbildung galt freilich immer als »unbolschewistisch«. Auch hier war der Keim des Neuen, die Ausrottung jeder Opposition, schon im Alten angelegt. Aber der Fraktionskampf beherrschte die Bühne der Kominternpolitik, und die russische Führung war daher keine eindeutige Größe.
\end{abstract}

Weiter schrieb Borkenau in diesem Zusammenhang in seinem Beitrag über die Komintern:

Die russischen Verhältnisse übertrugen sich auf die Kominternparteien. Jeder russische Fraktionskampf übertrug sich auf die außerrussischen kommunistischen Parteien. Aber es gab auch innere Kämpfe innerhalb dieser Parteien, die ihre Wurzel nicht in Rußland, sondern in den politischen Problemen der einzelnen Länder hatten. Die kommunistischen Parteien hatten ein mehr oder weniger demokratisches Innenleben. Innerparteiliche Diskussionen waren noch nicht wie heute bloßer Schein, zur Irreführung der einfachen Mitglieder und der Außenwelt bestimmt. [...] Da die russische kommunistische Partei selbst noch nicht totalitär war, konnte sie auch den nichtrussischen Parteien kein totalitäres Regime aufzwingen. ${ }^{380}$

379 Franz Borkenau, Die neue Komintern, in: Der Monat 1 (1949), H. 4, S. 50-60, hier S. 52.

380 Ebd., S. $52 f$. 
Wie bereits erwähnt, stand für Lenin und für Trotzki nach der bolschewistischen Revolution im »rückständigen« Russland fest, dass insbesondere der proletarischen Revolution in Deutschland eine alles entscheidende Rolle und Funktion beikommt. Naheliegenderweise war die Führung des bolschewistischen Regimes bestrebt, zum einen die KPD in »ihre « Hand zu bringen und zum anderen die Aufnahme vor allem der einflussreichen Führer der sogenannten Unabhängigen Sozialdemokratischen Partei Deutschlands (USPD) in die Komintern - wie zum Beispiel Rudolf Hilferding und Karl Kautsky - zu verhindern, weil man befürchtete, dass die politische und theoretische »Linie« von Lenin und Trotzki gefährdet werden könnte. Die KPD, die Anfang der 1920er-Jahre die stärkste kommunistische Partei in Europa war, geriet immer mehr in finanzielle und politische Abhängigkeit Moskaus. Nachdem die Mitglieder der KPD im Dezember 1920 unter anderem die "gemäßigten« Paul Levy und Klara Zetkin mit der Führung beauftragten, die der »Moskauer Exekutive« um Sinowjew nicht radikal genug waren, gelang es, ihre Absetzung zu erreichen. Die neue Führung der KPD zettelte auf Betreiben der Bolschewiki im März 1921 einen Aufstand in Deutschland an, der misslang und der für die Partei politisch verheerende Folgen hatte, denn er führte zu Massenaustritten und bitteren Wahlniederlagen. Nachdem auch im Oktober 1923 die in den Augen von Lenin unmittelbar bevorstehende Revolution in Deutschland in der Ära von Brandler und Thalheimer mit einem Desaster endete, übernahmen mit Arkadi Maslow und Ruth Fischer zwei Funktionäre die Führung der KPD, die im ständigen (Richtungs-)Streit mit Moskau lagen. Die Komintern wurde erst wieder zu einem genuinen politischen Instrument Moskaus, insbesondere der sowjetischen Innenpolitik, nachdem die neue Parteileitung mit tatkräftiger Unterstützung des Kremls gestürzt wurde und Ernst Thälmann mit der Führung der KPD beauftragt wurde. ${ }^{381}$

Mit dem Ausbleiben der erwarteten proletarischen Revolution - nicht erst im Jahre 1923 - vor allem in Deutschland wurde das bolschewistische Regime in Russland frühzeitig auf sich selbst zurückgeworfen. Nach dem Sieg im Bürgerkrieg und nach dem Kronstädter Aufstand wurde unter der Federführung Lenins die Liberalisierung des Wirtschaftslebens, sprich die Neue Ökonomische Politik (NEP), auf dem X. Parteitag im März 1921 ins Leben gerufen - es wurden Zugeständnisse an die private Wirtschaft gemacht, um Industrie, Handel und Agrarproduktion anzukurbeln; hierbei wurde sogar ausländisches Kapitel zugelassen -, die notwendig wurde, weil sich die Versorgungssituation nicht nur der städtischen Bevölkerung, sondern nicht zuletzt der Bauern im ganzen Land in einem katastrophalen Zustand befand und die Unzufriedenheit gegenüber der Sowjetregierung in den Augen der Machthaber ein bedrohliches Ausmaß angenommen hatte. ${ }^{382}$ Mit der NEP wurde unter anderem den Bauern, die durch den »Kriegskommunismus« vollkommen ausgeplündert wurden, die Möglichkeit eingeräumt, ihre Produkte gegen eine Besteuerung auf dem Markt frei zu verkaufen, was sozusagen den Beginn »kapitalistischer« Verhältnisse in Russland darstellte. Das Ergebnis der NEP war, dass sich die Lebensbedingungen in Russland erheblich verbesserten, ohne dass die Führung der Bolschewiki von ihrem Ziel der Einführung des Kommunismus, sprich: Sozialismus, abstand nahm. In den Augen von Löwenthal entwarf Lenin in seiner 1922 erschienenen »berühmten Schrift über Genossenschaftswesen« sein (politökonomie-)theoretisches Modell, »auf dem auch

381 Siehe ebd., S. $53 \mathrm{f}$.

382 Vgl. unter diesem Aspekt Ceorge Grosz, Russlandreise 1922, in: Der Monat 5 (1953), H. 56, S. 135-152. 
ein isoliertes Rußland eines Tages mit Hilfe einer weiterentwickelten Staatsindustrie eine kapitalistische Entwicklung der Landwirtschaft vermeiden und das flache Land in sozialistische Richtung transformieren könne ${ }^{383}$.

Zeitlich parallel zur Einführung der NEP brachte die leninsche Regierung eine andere Entwicklung zum Abschluss. Im Kern bestand Sowjetrussland bis zu diesem Zeitpunkt aus der großrussischen Sowjetrepublik, die bereits im Juli 1918 ihre erste Verfassung erhalten hatte und die mit den anderen kleineren Sowjetrepubliken - Ukraine, Weißrussland sowie den drei kaukasischen Republiken - ausschließlich durch Verträge verbunden war. Dieses Band wurde Ende Dezember 1922 fester geknüpft, denn auf dem X. Sowjetkongress wurde die Union der Sozialistischen Sowjetrepubliken (UdSSR) gegründet. Vor diesem Hintergrund spielte sich ein heftiger Streit zwischen Lenin und Stalin ab, der nach der Machtergreifung der Bolschewiki zum Leiter des Volkskommissariats für das Nationalitätenwesen ernannt wurde und noch wenige Monate zuvor, im April 1922, auf Vorschlag Lenins zum Generalsekretär der Partei gewählt wurde. Der Anlass war eine unterschiedliche Auffassung in Bezug auf das "Nationalitäten-Problem « - das betraf neben theoretischen Aspekten vor allem auch die tatsächliche politische Praxis, denn Lenin war entschieden gegen Stalins Unterdrückung der georgischen nationalen Opposition ${ }^{384}$-, die, wenn Lenin nicht im März 1923 einen neuerlichen Schlaganfall erlitten hätte und dann am 21. Januar 1924 verstorben wäre, zur Absetzung Stalins geführt hätte. ${ }^{385}$

Gleichzeitig mit der Verschlechterung des Gesundheitszustandes von Lenin infolge des ersten Schlaganfalls im Mai 1922, die für ihn das allmähliche Zurückziehen von der Leitung der Partei- und Staatsgeschäfte bedeutete, kam ein Kampf um die Macht zwischen Sinowjew, Trotzki und Stalin in Gang. Sinowjew war Präsident der Komintern und des Petrograder Sowjets, Trotzki Vorsitzender des militärischen Revolutionsrates und Kriegskommissar, Stalin hatte den einflussreichen Posten des Generalsekretärs der Partei inne. Während der Krankheit Lenins führte Stalin gemeinsam mit Sinowjew und Kamenew die Parteigeschäfte weiter. Innerhalb dieses Triumvirats, der sogenannten Troika, konnte Stalin seinen Einfluss erweitern, indem er seine beiden »Partner« auf der einen Seite und Trotzki auf der anderen Seite geschickt gegeneinander ausspielen konnte. Die Mitglieder des Zentralkomitees standen mehrheitlich hinter Sinowjew. Die sechs Mitglieder des Politbüros - der wichtigsten Machtinstanz zwischen Partei und Staat - waren bis auf Lenin erklärte Gegner Trotzkis, wobei allerdings der Gewerkschaftsführer Tomski keine zentrale Rolle spielte. Die übrigen Mitglieder, d. h. Sinowjew, Kamenew und Bucharin, bildeten sozusagen eine Koalition gegen Stalin. Vor dem Hintergrund des entfachten Machtkampfes zwischen Sinowjew, Trotzki und Stalin traf David Shub in seinem Beitrag Lenins letzte Tage im Monat mit Blick auf Sinowjew und Stalin die folgende zutreffende Feststellung:

383 Richard Löwenthal, Stalins Vermächtnis. Zur Interpretation seiner letzten Schrift, in: Der Monat 3 (1953), H. 55, S. 17.

384 Siehe Ruth Fischer, Tito und Trotzki. Der Unabhängigkeitskampf der kommunistischen Parteien, in: Der Monat 2 (1950), H. 16, S. 398-409, hier S. 399.

385 Vgl. zum »Bruch« zwischen Lenin und Stalin insgesamt Franz Borkenau, Der Spötter als Paneggyriker, in: Der Monat 3 (1951), H. 36, S. 606-615, sowie speziell zum »Nationalitäten-Problem« die S. 612-615. 
Aber weder im Zentralkomitee noch im Politbüro dachte die Mehrheit jemals daran, Sinowjew zum Führer zu erheben, da diese Führerrolle die Beherrschung des Parteikongresses zur Voraussetzung hatte. Und hier hatte Stalin das Heft in der Hand. Langsam brachte er seine Leute überall als Sekretäre in der Parteiorganisation unter und schreckte vor nichts zurück, um die Kontrolle des Parteiapparates in seine Hand zu bekommen. ${ }^{386}$

Aber auch wenn die Mitglieder des Politbüros mehrheitlich gegen Trotzki eingestellt waren und nicht daran dachten, Sinowjew zum späteren Nachfolger Lenins zu machen, war zum Zeitpunkt der Krankheit Lenins keineswegs klar, dass mehrere Jahre danach Stalin zum Alleinherrscher avancierte. Denn als Lenin am 21. Januar 1924 in seinem Landhaus in Gorki in der Nähe Moskaus verstarb, waren zweifelsohne »viele Wege« (Arendt) möglich.

\subsection{Der Aufstieg Stalins und die "Zweite Revolution« Ende der 1920er-Jahre}

Beim Tod Lenins war seine Nachfolge nicht genau geregelt. Allerdings lag das »Politische Testament « vom 25. Dezember 1922 vor, das seine Ansicht über die oberste Parteiführung in einer Übergangsform zum Ausdruck brachte. Angesichts der Gefahr einer möglichen Parteispaltung, die Lenin insbesondere aufgrund der starken Rivalität zwischen Stalin und Trotzki befürchtete, favorisierte er implizit eine kollektive Führung. Darüber hinaus bezeichnete er Bucharin einerseits als den »wertvollsten Theoretiker der Partei« und andererseits als den »Liebling der ganzen Partei«. In dem »Nachtrag《 vom 4. Januar $1923 \mathrm{zu}$ seinem »Testament" sprach sich Lenin auch angesichts von Stalins negativen Charaktereigenschaften klipp und klar für dessen Entfernung vom Posten des Generalsekretärs aus. Am 5. März 1923 brachte er Stalin persönlich in einem Schreiben das Ende der "persönlichen und kameradschaftlichen Beziehungen« zum Ausdruck. Genau schrieb Lenin in seinem »Politischen Testament«, das von David Shub in seinem Beitrag Lenins letzte Tage im Monat veröffentlicht wurde:

Genosse Stalin hat, nachdem er Generalsekretär geworden war, ungeheure Macht in seinen Händen vereinigt, und ich bin nicht sicher, ob er es immer versteht, von dieser Macht mit genügender Behutsamkeit Gebrauch zu machen. Andererseits zeichnet sich Cenosse Trotzki, wie sein Kampf gegen den Zentralausschuß im Zusammenhang mit der Frage des Volkskommissariats für das Verkehrswesen bewiesen hat, nicht nur durch außergewöhnliche Fähigkeiten aus - er ist sicherlich der fähigste Kopf im gegenwärtigen Zentralkomitee -, sondern auch durch übermäßiges Selbstvertrauen und durch die Neigung, sich zu sehr der rein administrativen Seite der Regierungsgeschäfte zu widmen.

Diese beiden Eigenschaften der beiden fähigsten Führerpersönlichkeiten im gegenwärtigen Zentralkomitee könnten ganz unwillkürlich zu einer Spaltung führen. Wenn unsere Partei keine Maßnahmen zu ihrer Verhinderung ergreift, könnte sie ganz unerwartet eintreten.

386 David Shub, Lenins letzte Tage. Aus einer neuen kritischen Biographie, in: Der Monat 1 (1948), H. 3, S. 45 . 